FRI0061

THE CONTRIBUTION OF TENOSYNOVITIS OF SMALL JOINTS TO THE SYMPTOM MORNING STIFFNESS IN PATIENTS PRESENTING WITH UNDIFFERENTIATED AND RHEUMATOID ARTHRITIS

Aleid Boer ${ }^{1}$, Debbie Boeters ${ }^{1}$, Ellis Niemantsverdriet ${ }^{1}$, Annette van der Helm - van Mil $^{1,2}$. ' LUMC, Reumatology, Leiden, Netherlands; ${ }^{2}$ ErasmusMC, Reumatology, Rotterdam, Netherlands

Background: Morning stiffness (MS) is characteristic of Rheumatoid Arthritis (RA) that is associated with functional disability. Despite the known parallel in the circadian rhythm of MS and that of several hormones and pro-inflammatory cytokines in the systemic circulation, it is insufficiently known to what extent local inflammatory processes contribute to this symptom. The correlation between MS and the number of swollen joints is relatively weak but may be underestimated by insufficient sensitivity in measuring local inflammation. $\mathrm{MRI}$ is more sensitive in detecting local inflammation. Furthermore, MRI-detected tenosynovitis of small joints is increasingly recognized as early feature of RA which is also associated with functional impairments. Recently it was proposed that this may contribute to MS.

Objectives: We assessed the relationship between MS and MRI-detected inflammation and tenosynovitis in particular.

Methods: 286 consecutive patients newly presenting with undifferentiated and rheumatoid arthritis underwent contrast-enhanced 1.5T-MRI of (2-5) MCP-, (1-5)MTP-, and wrist-joints. Scans were scored for tenosynovitis according to Haavardsholm and for synovitis, osteitis conform the RAMRIS-method. MS was dichotomized as $>60$ minutes. Associations between MS and tenosynovitis/synovitis were tested with logistic regression and the presence of a biologic interaction was assessed categorically (solitary or simultaneous presence of synovitis/tenosynovitis).

Results: MS was present in $40 \%$ of patients. Tenosynovitis was more often present in patients with MS than without MS (80\% versus $65 \%$ ), OR $2.11(95 \% \mathrm{Cl} 1.21 ; 3.69)$. Also synovitis was more often present in patients with MS (58\% versus 44\%), OR 1.83 (1.12;2.96). In categorized analyses the largest association was found for concurrent synovitis and tenosynovitis OR $2.43(1.30 ; 4.54)$; whereas single presence of synovitis was not associated (OR $0.85(0.21 ; 3.47)$ ). The variance explained in all analyses on morning stiffness was small, ranging $3-6 \%$.

Conclusion: Tenosynovitis, and simultaneous presence of tenosynovitis and synovitis in particular, was associated with MS. However, effect sizes suggested that the contribution of local inflammation to this symptom is rather limited.

Acknowledgement: E.C. Newsum and W.P. Nieuwenhuis are acknowledged for scoring MRI-scans.

Disclosure of Interests: Aleid Boer: None declared, Debbie Boeters: None declared, Ellis Niemantsverdriet: None declared, Annette van der Helm van Mil Grant/research support from: The research leading to these results has received funding from the European Research Council (ERC) under the European Union's Horizon 2020 research and innovation programme (Starting grant, agreement No 714312) and from the Dutch Arthritis Foundation.

The funding source had no role in the design and conduct of the study. DOI: 10.1136/annrheumdis-2019-eular.1782

\section{FRI0062 INFECTIONS AMONG RHEUMATOID ARTHRITIS PATIENTS STARTING OR SWITCHING BIOLOGICAL AGENTS. A SYSTEMATIC LITERATURE REVIEW}

Marta Novella-Navarro ${ }^{1}$, Alejandro Gomez-Gomez ${ }^{2}$, Maria Auxiliadora MartinMartinez ${ }^{3}$, Mercedes Guerra-Rodriguez ${ }^{3}$, Enrique Raya ${ }^{4} .{ }^{1}$ Hospital Universitario de Torrejon, Rheumatology, Madrid, Spain; ${ }^{2}$ Hospital Universitario Infanta Sofía, Rheumatology, Madrid, Spain; ${ }^{3}$ Spanish Society of Rheumatology, Research Unit, Madrid, Spain; ${ }^{4}$ Hospital Universitario San Cecilio, Rheumatology, Granada, Spain

Background: The increased rate of infections is one of the most relevant issues regarding biologic therapies in Rheumatoid Arthritis (RA). There is a large number of studies comparing different biologic agents and biologic vs synthetic DMARDs in order to assess their safety profile. Nevertheless, the main question is whether biologics really increase the infection risk by themselves. Up to date, different studies tried to clarify the actual role that these drugs have in this potentially serious adverse effect, searching for other predisposing conditions related to patient clinical characteristics.

Objectives: To compare the risk of infections between patients starting biologic for the first time (naïve) and patients with an inadequate response to a previous biologic agent (switchers)

Methods: A search strategy was performed by the Spanish Society of Rheumatology documentalist over Pubmed, Embase and Cochrane Library databases (from January 2008 to February 2018). All the references retrieved were managed using Endnote $X 7^{\circledR}$. Screening of studies, data collection and data analysis was performed by 2 reviewers. Meta-analysis, systematic reviews of Randomized Controlled Trials (RCT), Clinical Trials or Cohort studies, comparing data from adult RA patients starting biolog ics and switching biologics, were selected. After the first screening, a critical review was performed using electronic FLC platform for critical appraisal tools $\left(\mathrm{Osteb}^{\circledR}\right)$ and The Scottish Intercollegiate Guidelines Net work (SIGN) was considered to classify the scientific evidence of the selected studies. Studies including patients with chronic infections, tuberculosis reactivations or surgical infections were excluded.

Results: 8 studies were included: 1RCT, 2 Open label study, 1 Open label extension from RCT, 2 subgroups analysis from RCTs and 2 prospective cohort studies.

Overall infection rate was higher in switchers than in naïve patients, with a mean variation between $0-9$ and 1.8100 PY, serious infections were also higher in switchers patients, 2.2 100PY. In both groups, (naïve and switchers), patients treated with more cumulative dose of glucocorticoids (GC) had an increased risk of infections, and it was higher in patients with an inadequate response to previous biologic agents. Mean disease duration was longer in switchers, but this fact was not related to increase of infections.

Conclusion: This systematic review show that overall rate of infections is slightly higher in switchers than in naïve patients, but the fact of having received several biologic agents does not present itself as a risk factor for infection. However, the most important risk factor in the development infections was the higher cumulative doses of GC.

Disclosure of Interests: None declared

DOI: 10.1136/annrheumdis-2019-eular.3175

\section{FRI0063 \\ EFFECTS OF MALALIGNMENT AND DISEASE ACTIVITY ON SECONDARY OSTEOARTHRITIS PROGRESSION IN KNEES OF RHEUMATOID ARTHRITIS PATIENTS}

Noriaki Okumura, Taku Kawasaki, Kosuke Kumagai, Mitsuhiko Kubo,

Takafumi Yayama, Tomohiro Mimura, Tsutomu Maeda, Shinji Imai. Shiga

University of Medical Science, Orthopaedics Surgery, Otsu, Japan

Background: Recent advancements in treatment of rheumatoid arthritis (RA) with disease-modifying anti-rheumatic drugs (DMARDs) have been remarkable, with disease symptoms nearly disappearing due to their strong anti-inflammatory action and many patients achieving remission. As a result, the need for RA-related surgery has shown a yearly decreasing trend, especially knee surgery and synovectomy procedures [1]. On the other hand, cases of secondary osteoarthritis $(\mathrm{OA})$ in knee joints as a symptom associated with RA following a total knee arthroplasty (TKA) are increasing.

Objectives: We investigated the morphology of osteophytes by quantitatively evaluating their size using images obtained prior to performing a TKA. Additionally, the relationships of osteophyte size with patient background, disease activity, and degree of inflammation were examined.

Methods: Radiographs of 35 consecutive knees in 30 RA patients (26 females, 4 males; mean age 63.0 years; median disease duration 15 years) who underwent TKA, including preoperative standing AP view radiographs of the knee joint, were retrospectively analyzed. Using the Image-J software package, osteophyte size in the medial femur (MF), medial tibia (MT), lateral femur (LF), and lateral tibia (LT) regions was determined. Written informed consent for data collection was obtained from all patients in accordance with the Declaration of Helsinki.

Results: Preoperative Larsen grade was 2, 3, 4, and 5 in 1, 12, 18 and 2 patients, respectively, while the mean range of motion of the knee joint was $118^{\circ}$ for flexion and $-10^{\circ}$ for extension. The mean femorotibial angle (FTA) was $178 \pm 13.6^{\circ}$, with varus $\left(\mathrm{FTA}>180^{\circ}, \mathrm{n}=14\right)$ more frequently observed as compared to valgus (FTA $<170^{\circ}, n=7$ ) cases. Mean osteophyte size in the MF, MT, LF, and $\mathrm{LT}$ regions was $37.2,17.0$ 27.2, and $4.57 \mathrm{~mm}^{2}$, respectively, and significantly greater in the medial compartment $(M F+M T)$ than the lateral compartment $(L F+L T)(p<0.001)$. In the varus cases, osteophyte size in the medial compartment was significantly larger as compared to the normal and valgus cases $(p=0.0016)$ Furthermore, osteophyte size in the medial compartment was negatively correlated with the inflammatory markers CRP $(r=-0.492, p=0.0027)$ and ESR ( $r=-0.529, p=0.0016)$, whereas that in the lateral compartment was negatively correlated with disease activity $(r=-0.589, p=0.0023)$.

Conclusion: Our results suggest that secondary $\mathrm{OA}$ is a more prominent symptom in RA patients in whom inflammation is controlled, while disease activity has effects on osteophyte size. 